\title{
Experimental characterisation of leak through elastomer-metal interface
}

\section{Kambhammettu Sri Krishna Sudhamsu* and C. Lakshmana Rao}

Department of Applied Mechanics, Indian Institute of Technology Madras, Chennai - 600036, India

Email: ksksudhamsu@gmail.com

Email: lakshman@iitm.ac.in

*Corresponding author

\author{
Abhijit P. Deshpande \\ Department of Chemical Engineering, \\ Indian Institute of Technology Madras, \\ Chennai - 600036, India \\ Email: abhijit@iitm.ac.in
}

\section{Jithin Devan}

Department of Applied Mechanics, Indian Institute of Technology Madras, Chennai - 600036, India Email: jithin_devan@yahoo.com

\begin{abstract}
Elastomeric seals are devices that are widely used to prevent fluid leakage through the interface of mating parts. In this paper, we study the leak characteristics of a representative elastomeric seal system using an experimental setup that has been developed to measure the leak rate of gas through an elastomer-metal interface as a function of sealing load intensity and fluid pressure. These experiments are carried out on nitrile butadiene rubber (NBR), hydrogenated nitrile butadiene rubber (HNBR) and fluoro-elastomer (FKM) specimens using nitrogen gas at pressures ranging from $40 \mathrm{kPa}$ to $800 \mathrm{kPa}$. The experiments revealed that the leak rate increases rapidly with gas pressure and decreases with sealing load intensity. When leak rate was plotted against normalised gas pressure, it was observed that all the data points fall reasonably on one single curve irrespective of sealing load intensity and the material. These results will be useful for further analysis in developing a mathematical model for characterising fluid leak through elastomer-metal alloy interfaces.
\end{abstract}

Keywords: elastomeric; seals; leak; failure; prediction of leak; elastomer-metal interface; modelling; experimentation.

Reference to this paper should be made as follows: Sudhamsu, K.S.K., Rao, C.L., Deshpande, A.P. and Devan, J. (2019) 'Experimental characterisation of leak through elastomer-metal interface', Int. J. Materials and Structural Integrity, Vol. 13, Nos. 1/2/3, pp.186-199. 
Biographical notes: Kambhammettu Sri Krishna Sudhamsu is a Doctoral student in the Department of Applied Mechanics, Indian Institute of Technology Madras. He obtained his BTec degree from the Indian School of Mines, India. His research interests include failure prediction of seals and impact and blast resistance of structures.

C. Lakshmana Rao is a Professor in the Department of Applied Mechanics at Indian Institute of Technology Madras. He obtained his BTech and MS degrees from the IIT Madras and $\mathrm{ScD}$ degree from the Massachusetts Institute of Technology. He has authored three books namely Engineering Mechanics Statics and Dynamics, Modelling of Engineering Materials and Applied Impact Mechanics. His research interests include characterisation of smart materials, fracture assessment of structures, impact and blast resistance and failure prediction of seals.

Abhijit P. Deshpande is a Professor in the Department of Chemical Engineering at Indian Institute of Technology Madras. He obtained his BTech degree from the IIT Bombay, MS degree from the University of Pittsburgh and $\mathrm{PhD}$ from the University of Washington. He teaches courses on Continuum Mechanics, Polymer Rheology, Momentum Transfer and Thermodynamics. His research interests include polymer rheology, flow visualisation, ionically conducting polymers and polymeric composites.

Jithin Devan is a Master's degree student at the Department of Applied Mechanics, Indian Institute of Technology Madras. He obtained his BTech degree from the Manipal institute of technology, India. His research interests include finite element analysis and simulation of failure in seals.

This paper is a revised and expanded version of a paper entitled 'Leak characteristics of elastomeric seals' presented at Indian Conference on Applied Mechanics, MNNIT Allahabad, 7 July 2017.

\section{Introduction}

Seals are devices that are used to prevent passage of fluid through the interface of two mating parts. Their presence can be seen in various industrial and household applications such as those in cryogenic liquid tanks, solid rocket boosters, oil well completion equipment, etc. Seals are made using a wide range of materials like metal alloys, elastomers, thermoplastics, etc. Seals that are made of elastomers are known as elastomeric seals. Elastomeric seals have been in use for prevention of fluid leaks since the invention of O-ring in the late half of nineteenth century (Druecke et al., 2015). Since then, elastomeric seals have found their way into various applications in sealing liquids and gases. Today, these seals are used to seal liquid and gaseous pressures ranging from few kilopascals (as in airtight containers) to tens of thousands of kilopascals (as in oil and gas production) at temperatures ranging from $-200^{\circ} \mathrm{C}$ (while handling cryogenic liquids) to $200^{\circ} \mathrm{C}$ (in sealing oil and gas production fluids) (Weitzel et al., 1960; Ito et al., 2011). Figure 1 shows the cross-section view of a typical elastomeric seal installed to prevent fluid from flowing out of the pressure chamber. The components of a sealing system such as elastomeric seal, seal-envelope interface, etc., are indicated in this figure. 
In all the years since their invention, research on elastomeric seals has been primarily on estimating and preventing failure of a sealing system (Gillen et al., 2003; Briscoe et al., 1994; Mars and Fatemi, 2002; Liu et al., 2017; Grelle et al., 2017). Failure of a sealing system (simply called 'seal failure' or 'failure of a seal' in this document) is said to have occurred when the fluid starts to leak through the seal or through the seal-surrounding envelope interface at a rate more than a predefined acceptable limit. In this paper, we adhere to this definition of failure. Leak through interface of any two surfaces can occur in different environments and loading conditions due to different reasons and researchers have separately devised methods to predict failures in each of these cases. For example, leakage of high pressure fluid through pipes of nuclear power plants is studied as a crack propagation problem and the pipes are designed to leak before breaking (Moan et al., 1990; Takahashi, 2002; Kirthan et al., 2016).

Figure 1 Elastomeric seal illustration and nomenclature (see online version for colours)

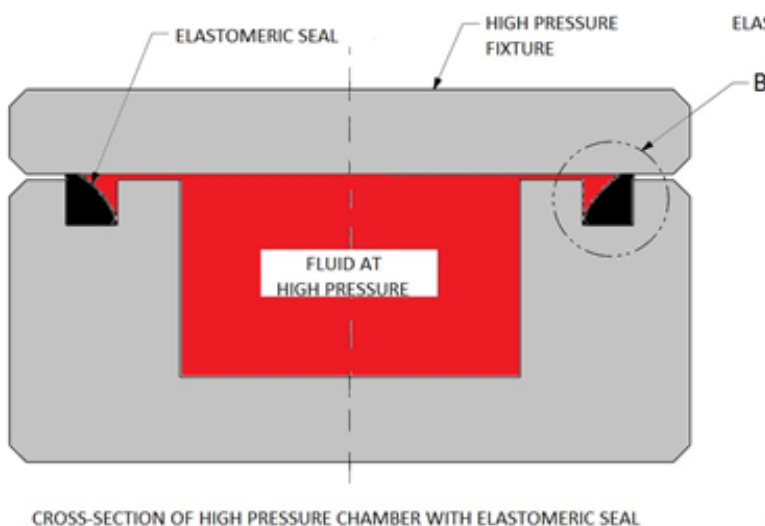

CROSS-SECTION OF HIGH PRESSURE CHAMBER WITH ELASTOMERIC SEAL

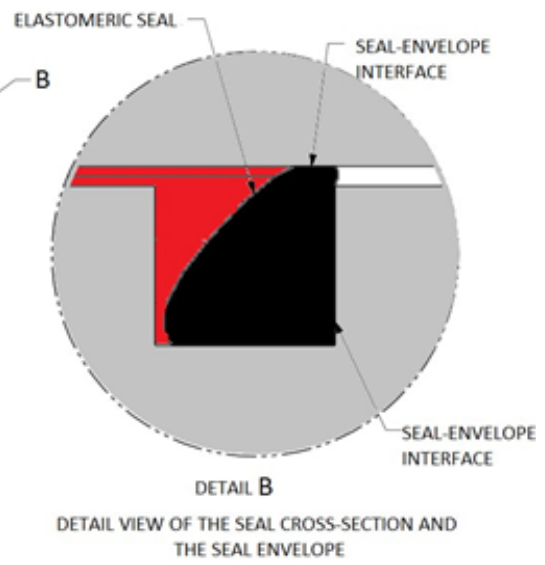

However, leak and failure in elastomeric seal systems due to corrosive environments and prolonged service time is studied as a material degradation problem using accelerated aging tests (Gillen et al., 2003; Liu et al., 2017; ISO 23936-2, 2011). Again, estimation of leak and seal failure that occurs due to a specific loading pattern known as rapid gas decompression (RGD) is carried out using different set of techniques (Briscoe et al., 1994; ISO 23936-2, 2011). In the next subsection, we will describe how failure of elastomeric seals is predicted in each of the above cases. We will then look into how the experiments that we have conducted to monitor leakage of gas through the interface between the elastomeric sheet and the metal can help in complementing the existing failure prediction/prevention procedures.

\subsection{Seal failure due to material degradation}

Seal failure due to degradation in the mechanical properties of elastomer with time is one of the most well explored areas in elastomeric seals (Gillen et al., 2003; Liu et al., 2017; Gillen et al., 2005; Etsion and Front, 1994). This degradation, referred to as 'aging', is even faster when the seal functions at high temperatures and/or in the presence of incompatible fluids that break the polymer chains or their crosslinks (Gillen et al., 2003; ISO 23936-2, 2011). The life of seals in such environments is generally estimated using 
accelerated aging tests (Liu et al., 2017; Moan et al., 1990; Gillen et al., 2005) that are conducted on the seals or on the elastomeric specimen. ISO 23936-2 (2011) is the standard used for life estimation of elastomeric seals that come into contact with oil and gas production media. The oil and gas production media typically consist of alkanes, cycloalkanes and aromatic hydrocarbons along with other compounds with elements like nitrogen, oxygen and sulphur. According to this standard, elevated fluid and environment temperature degradation tests are conducted on elongated elastomeric specimen and the change in a 'representative material property' is monitored with time. This representative material property is the mechanical property of the material that manufacturers consider critical for the sealing system to function. Once this property goes below the threshold, the seal is considered to fail.

Researchers have for long performed elevated temperature degradation tests directly on seals instead of material specimens. They considered that the contact load between the seal and its envelope (referred to as 'sealing load' from now on in this paper) at a given fluid pressure as a measure of sealing ability of the seal (Lorenz and Persson, 2009; Liu et al., 2014; Gillen et al., 2005). As material degrades, this sealing load drops and when this sealing load drops below a certain value (known as 'threshold sealing load'), the seal is estimated to leak.

\subsection{Seal failure induced by loading patterns}

In some very specific kinds of loading, leak can occur due to form failure of seal bodies (Briscoe et al., 1994; Liu et al., 2014). RGD is one example of a special loading observed in oil and gas applications where seal can develop cracks due to sudden reduction in gaseous sealing fluid pressure (Briscoe et al., 1994; ISO 23936-2, 2011). While operating at high pressure, the gas can diffuse and dissolve into the elastomer. When the pressure is reduced, this gas effuses back out of the elastomer. Sudden reduction in gas pressure induces high rates of effusion which in turn causes high tensile stresses and hence cracks inside the seal body (Briscoe et al., 1994). These cracks compromise the structural integrity of the elastomer and hence, its sealing ability (Grelle et al., 2017). ISO 23936-2 (2011) also discusses the qualification tests needed to be done to certify a seal material as RGD resistant for oil and gas applications. These tests are performed on an O-ring of predefined dimensions and depending on the size and number of cracks that the O-rings develop during testing; the material is given a performance rating.

In both the failure prediction methods for modes of failure discussed above, we observe that the relationships between the seal failure and the indicators of such failure such as sealing load, number of cracks, etc., are material and geometry specific. Hence, their seal failure prediction capability across seals of various shapes and sizes is limited. A fundamental geometry independent approach that can relate seal failure and indicators of such failure can complement the existing prediction techniques by overcoming this limitation. Liu et al. (2014) attempted this by modelling the mechanics of leak through the seal-envelope interface. The numerical model thus, developed considers that leak would happen when the interface separates due to the pressure loading of the seal and the envelope with the sealing fluid. To develop such models, an understanding of how leak varies with parameters such as sealing load intensity, fluid pressure, material properties and interfacial properties is needed. Such insight can be obtained by experiments that allow measurement of leak as any of the above parameters are varied. 
Liu et al. (2014) conducted experiments to estimate leak of water through the interface of hydrogel and acrylic sheets for different water pressures and sealing load intensities. The hydrogel was placed in between two acrylic sheets and sealing load was generated by compressing the hydrogel. The water pressure was varied up to $40 \mathrm{kPa}$ at three different compressions of $10 \%, 20 \%$ and $30 \%$, in the hydrogel. The results showed that the leak initiation pressure (smallest pressure at which leak can occur) increases with increase in the amount of compression or sealing load intensity. Liu, then, hypothesised that leak occurs whenever the seal loses contact with the sealing envelope.

Lorenz and Persson (2009) also conducted similar experiments to measure leak rate of water through the interface of an annular rubber ring and a rough substrate for different sealing load intensities and the surface roughness of the substrate at a constant water pressure of $10 \mathrm{kPa}$. The results obtained showed exponential drop in leak rate with increase in sealing load intensity. Some variation in leak rate with the surface roughness was also observed.

Both Lorenz and Persson's (2009) and Liu et al.'s (2014) experiments have given a valuable insight and information on how seals would behave with varying input parameters. At the same time, we also note that both performed experiments each on one seal material only at much lower pressures than those used in many industries like oil and gas, aerospace, automobile, pneumatic/hydraulic machinery, etc.

From an engineering perspective, we believe that, whenever possible, it is useful to conduct fundamental experiments that are aimed at capturing the mechanics of seal failure with sealing materials and environmental/loading variables similar to those experienced in the application. The insight and data generated by such experiments can be later used not only to verify the existing models but also to propose new models that can efficiently predict failure for specific applications. Keeping this in view, we have developed an experimental setup to study leak rate of a representative elastomeric seal as a function of sealing load intensity and fluid pressure. These experiments are carried out on nitrile butadiene rubber (NBR), hydrogenated nitrile butadiene rubber (HNBR) and fluoro-elastomer (FKM) sheet specimen using N2 gas at pressures ranging from $40 \mathrm{kPa}$ to $800 \mathrm{kPa}$.

In the next sections of this paper, the experiment and its results are discussed in detail. As discussed in this section, there is a need to relate seal failure (i.e., leak rate being above the threshold limit) and other evaluable parameters like sealing load intensity, fluid pressure, etc. The objective of this paper is to understand the relationship between leak rate, fluid pressure and sealing load intensity of leak through the elastomer-metal interface for NBR, FKM and HNBR elastomeric specimen through experimentation.

\section{Experimental details (materials and methods)}

Figures 2(a) and 2(b) show the schematic of the test fixture's cross section and the complete setup of the experiment. The test fixture contains a top die and a bottom die made of SS-316, an elastomeric sheet and containment seal as shown in Figure 2(a). The elastomeric sheet is sandwiched between the top and bottom dies as shown in Figure 2(a). 
The entire assembly is compressed using a displacement controlled compression testing machine (CTM) as shown in Figure 2(b) and gas pressure (nitrogen) is applied through the inlet port. Sealing occurs at the interface of the top die and the elastomeric sheet as shown in Figure 2(a). The containment seal routes the leaked gas into the leakage measurement port. The volume of the gas that leaks through this interface in a given time is measured and then pressure is increased. The leak volume is measured again and then the pressure is increased for another reading. This procedure is continued until the leak rate becomes too high to be measured $(>100 \mathrm{ml} / \mathrm{sec})$. The pressure is now bled and the elastomer is compressed even more before the pressure is applied again for a new set of measurements.

The entire experiment is repeated three times each time with a different sample of the same material to check the consistency in results. These experiments were conducted on three different elastomers namely NBR, HNBR and FKM. The NBR samples used $(\Phi 60 \mathrm{MM} \times 6 \mathrm{MM} \mathrm{THK})$ were taken from a commercial grade 70 shore A durometer elastomeric sheet. The FKM and HNBR samples $(\Phi 60 \mathrm{MM} \times 4 \mathrm{MM}$ THK) were trade named Technoflon manufactured by Solvey, Italy and Zeptol manufactured by Zeon, Japan, respectively.

Figure 2 Test fixture and experimental setup (see online version for colours)

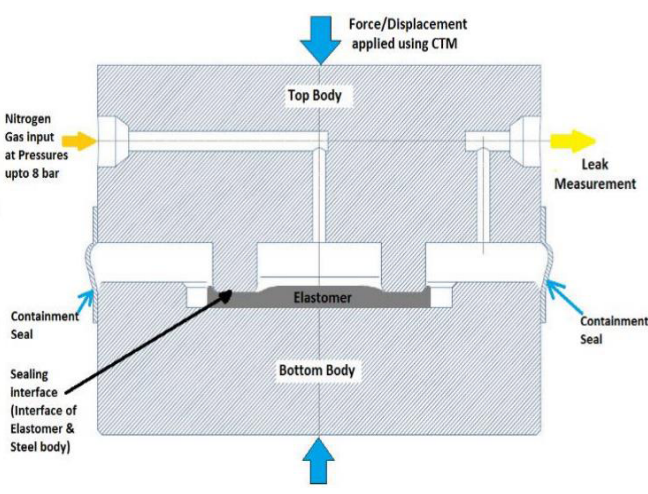

(a)

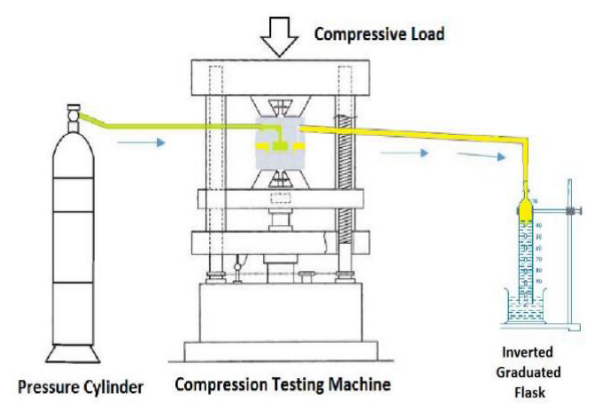

(b)

\section{Results}

Figures 3, 4 and 5 show how the leak rate of gas varied with applied gas pressure for the NBR, HNBR and FKM test specimens, respectively. Each curve is drawn at a different amount of compression and the corresponding sealing load intensity at zero pressure (sealing load at zero gas pressure divided by the interface area) is shown by the labels adjoining the curves. For all the three materials, we can notice that leak rate first increases slowly and then increases rapidly with gas pressure. With sealing load intensity at zero pressure, the leak rate decreases for all the three materials. 
Figure 3 Measured leak rates as function of gas pressure, contact pressure for each set of measurement is shown next to the leak rate curve for NBR specimen

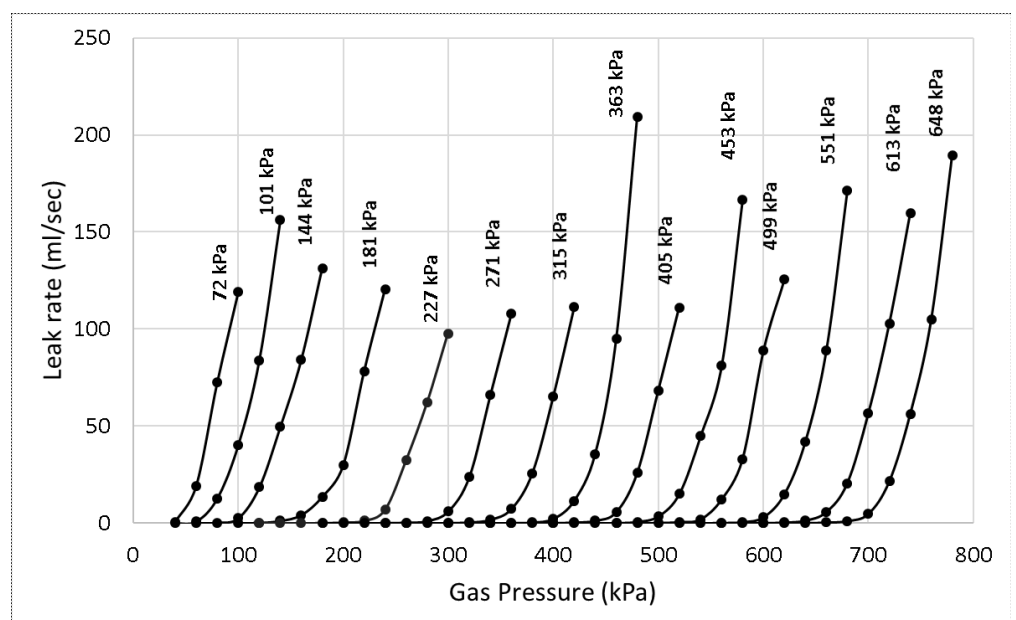

Figure 4 Measured leak rates as function of gas pressure, contact pressure for each set of measurement is shown next to the leak rate curve for HNBR specimen

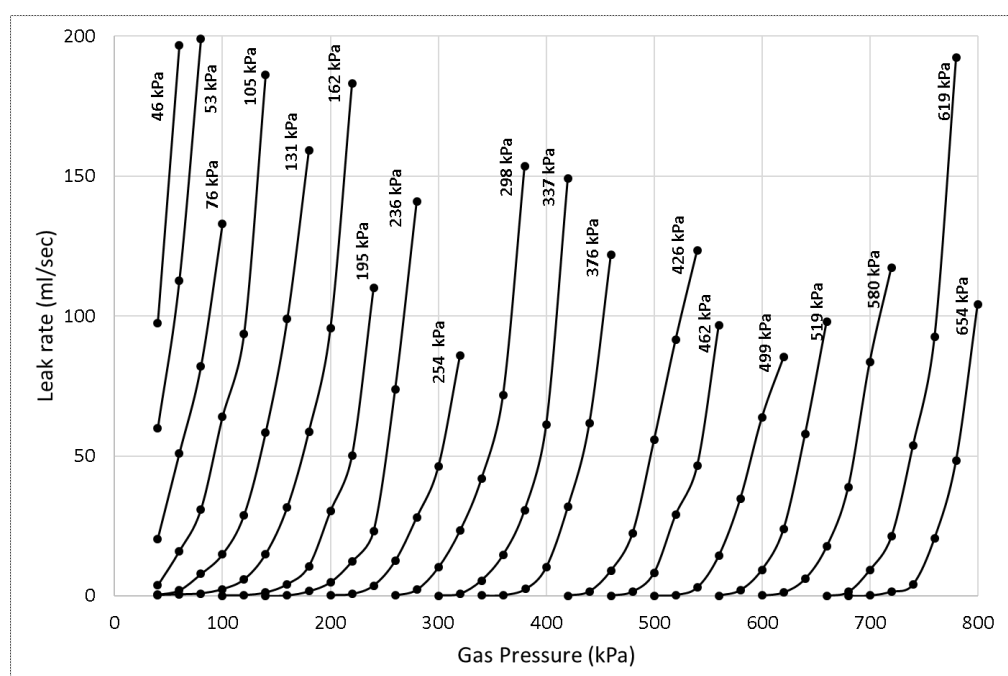

\section{Discussion}

In Figures 3-5, each curve can be extrapolated to intersect the x-axis. For example, in Figure 4, when the curve with sealing load intensity at zero pressure $105 \mathrm{kPa}$ is extrapolated, it meets $\mathrm{x}$-axis at $33 \mathrm{kPa}$ of gas pressure. It can be understood that any measurable leak starts at this gas pressure that corresponding to the extrapolated point 
and that this gas pressure is the leak initiation pressure. The first measurement point ( $40 \mathrm{kPa}$ of gas pressure in case of curve in Figure 4 with $105 \mathrm{kPa}$ sealing load intensity at zero pressure) can also be used for leak initiation pressure. However, this measurement would depend on the limits of the measuring equipment. Therefore, extrapolation of multiple points was used in this work as a measure of leak initiation pressure. Figures 6-8 show the leak initiation pressure as a function of sealing load intensity at zero pressure for two samples of NBR, HNBR and FKM. It can be observed that at any given sealing load intensity at zero pressure, FKM has a greater leak initiation pressure when compared to HNBR or NBR. For example, at sealing load intensity at zero pressure of $360 \mathrm{kPa}$, FKM specimen exhibits a leak initiation pressure $520 \mathrm{kPa}$ while NBR and HNBR show leak initiation pressures of $300 \mathrm{kPa}$ and $340 \mathrm{kPa}$, respectively. A similar insignificant difference between the leak initiation pressures exhibited by NBR and HNBR can be seen at other sealing load intensities at zero pressure as well. However, we note that direct comparison between the leak characteristics of NBR and other two material specimens cannot be done as the thickness of NBR specimen $(6 \mathrm{~mm})$ is different from those of FKM and HNBR specimen (4 mm). The differences in leak initiation pressures exhibited by different materials may be attributed to the differences in their interfacial properties and the details of such are to be investigated.

Figure 5 Measured leak rates as function of gas pressure, contact pressure for each set of measurement is shown next to the leak rate curve for FKM specimen

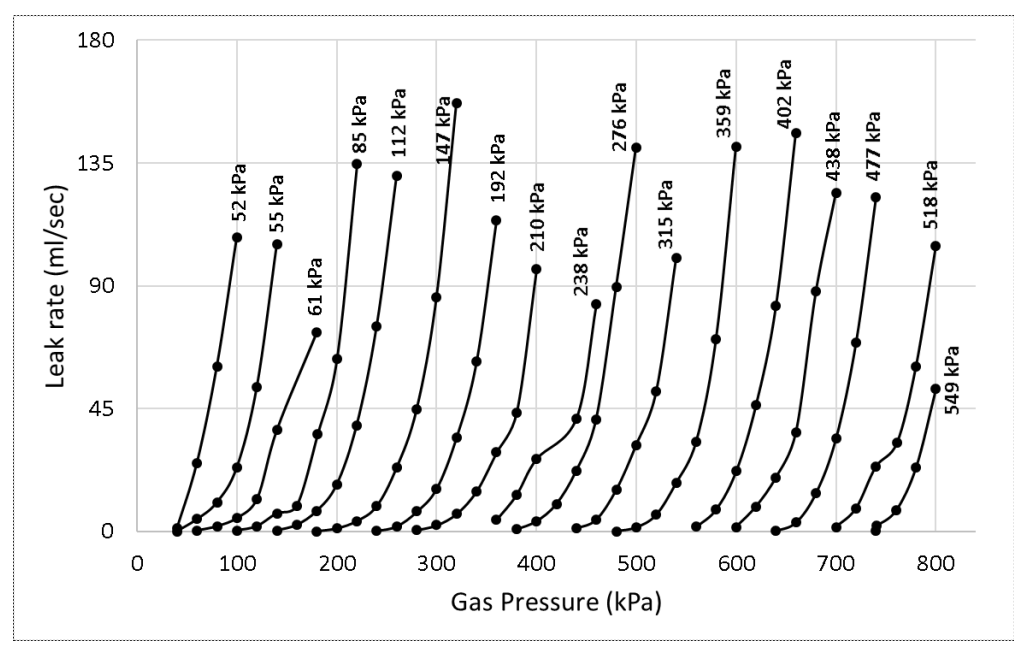

We note that one can select a leak threshold for failure and find the 'failure initiation gas pressure' (gas pressure at which seal fails) at various sealing load intensities. Figure 9 shows failure initiation gas pressure as a function of sealing load intensity for NBR, HNBR and FKM for a leak threshold of $20 \mathrm{ml} / \mathrm{sec}$. It can be observed that while FKM shows higher failure initiation pressure when compared to HNBR and NBR, NBR and HNBR do not show significant difference in their failure initiation pressures. This is similar to what has been observed with leak initiation pressure. 
Figure 6 Leak initiation pressure v/s sealing load intensity at zero pressure (NBR specimen) (see online version for colours)

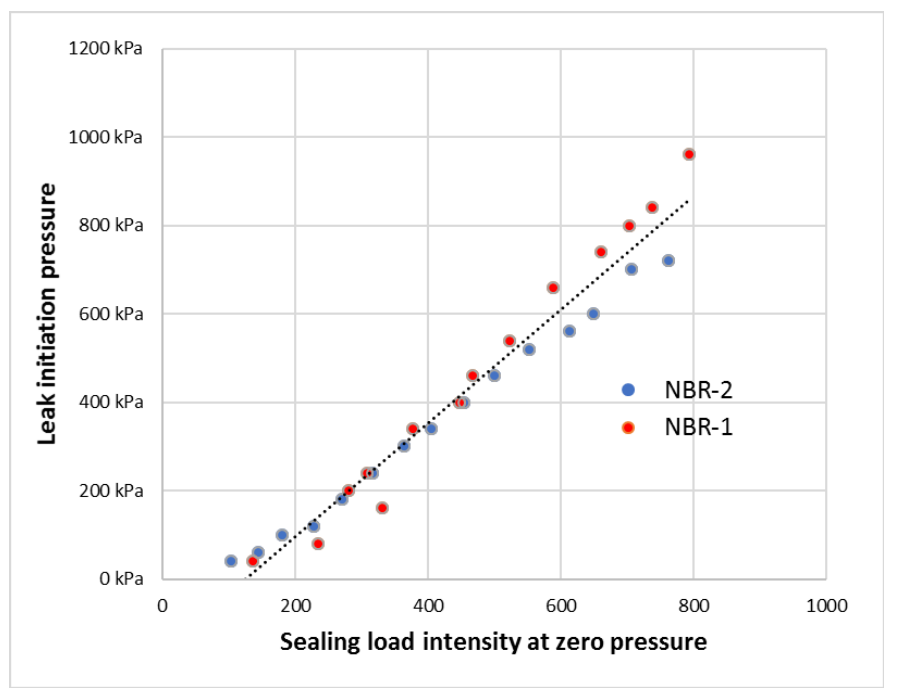

Figure 7 Leak initiation pressure v/s sealing load intensity at zero pressure (HNBR specimen) (see online version for colours)

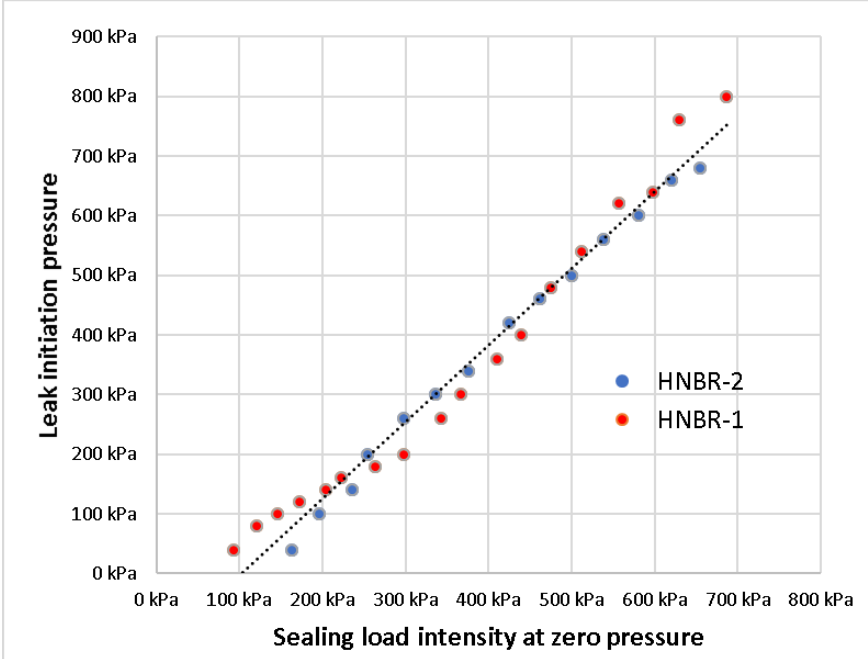

The gas pressures in Figures 3, 4 and 5 are normalised and the data points are re-plotted in Figures 10, 11 and 12. For every curve, gas pressures corresponding to a leak rate of $2 \mathrm{ml} / \mathrm{sec}$ and $50 \mathrm{ml} / \mathrm{sec}$ are linearly scaled to 0 and 1, respectively. It can be noted from these graphs that irrespective of sealing load intensity at zero pressure, all the points lie reasonably on the same curve. When the data points of all the three materials 
are plotted together on a single graph (as shown in Figure 13), it can be seen that all of them lie on same curve irrespective of material. We note that while leak initiation pressure curves characterise the material dependent behaviour of leak, the normalised curve characterises the material independent behaviour of the same leak.

Figure 8 Leak initiation pressure v/s sealing load intensity at zero pressure (FKM specimen) (see online version for colours)

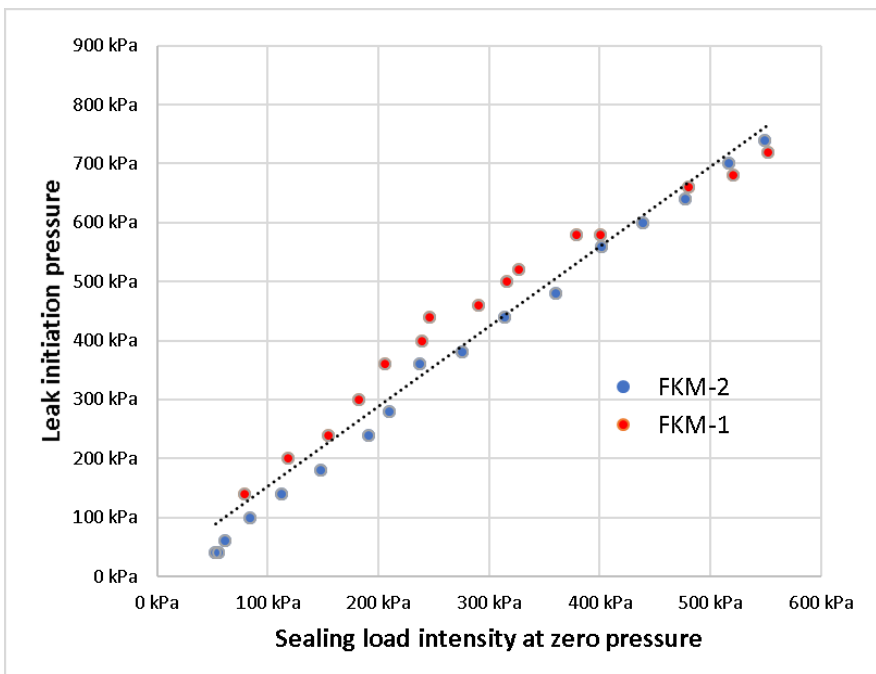

Figure 9 Failure initiation pressure (with leak threshold $20 \mathrm{ml} / \mathrm{sec}$ ) v/s sealing load intensity at zero pressure

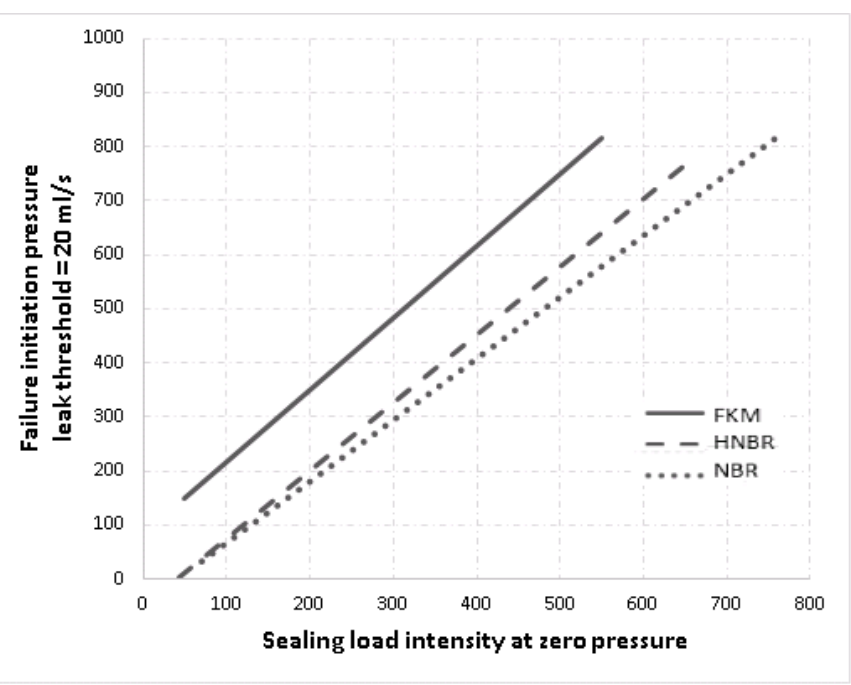


Figure 10 Leak rate v/s normalised gas pressure (NBR specimen) (see online version for colours)

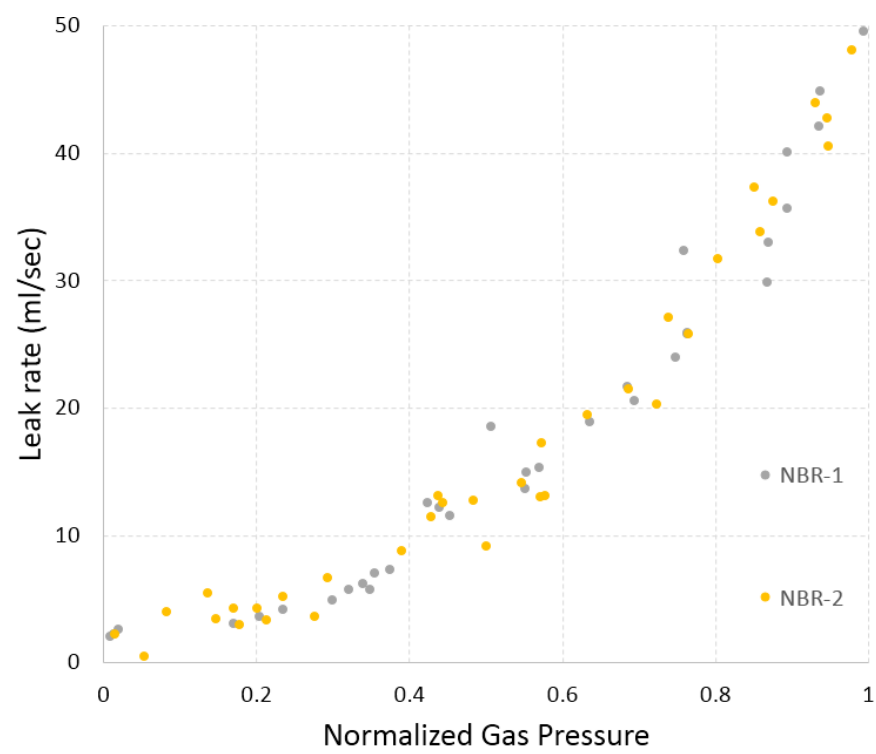

Figure 11 Leak rate v/s normalised gas pressure (HNBR specimen) (see online version for colours)

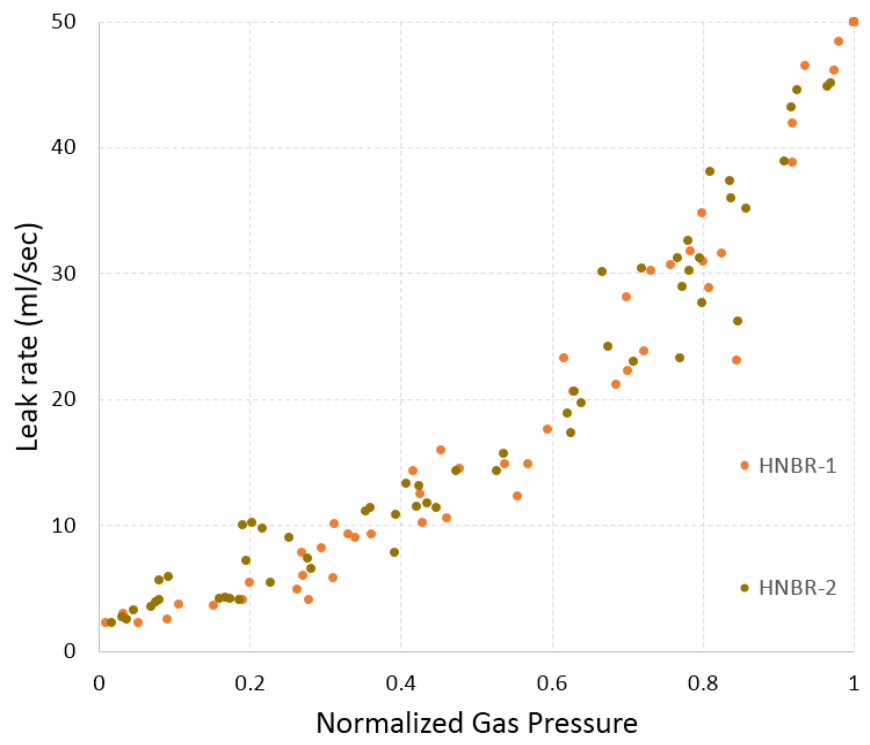

At the same time, we note that such a curve can be geometry dependent if not material dependent, and may not be useful to estimate leak in seals of different geometries. For predicting leak and failure of seals, a robust yet simple geometry and material independent model is required. Liu's model is an attempt made in this direction. The limitation of this model is that it does not consider the effects of interfacial properties such as adhesion and roughness of seal-envelope interface on leak. The authors suggest 
that such interfacial properties can be accounted for by using well developed literature in damage and fracture mechanics where separation at the interface can be considered analogous to crack propagation at the interface. Once such a theory is developed, the model can be calibrated using data from experiments such as the ones reported in this work. This model can then be used to predict leak through the interface of seals of different sizes and shapes such as O-rings and S-seals.

Figure 12 Leak rate v/s normalised gas pressure (FKM specimen) (see online version for colours)

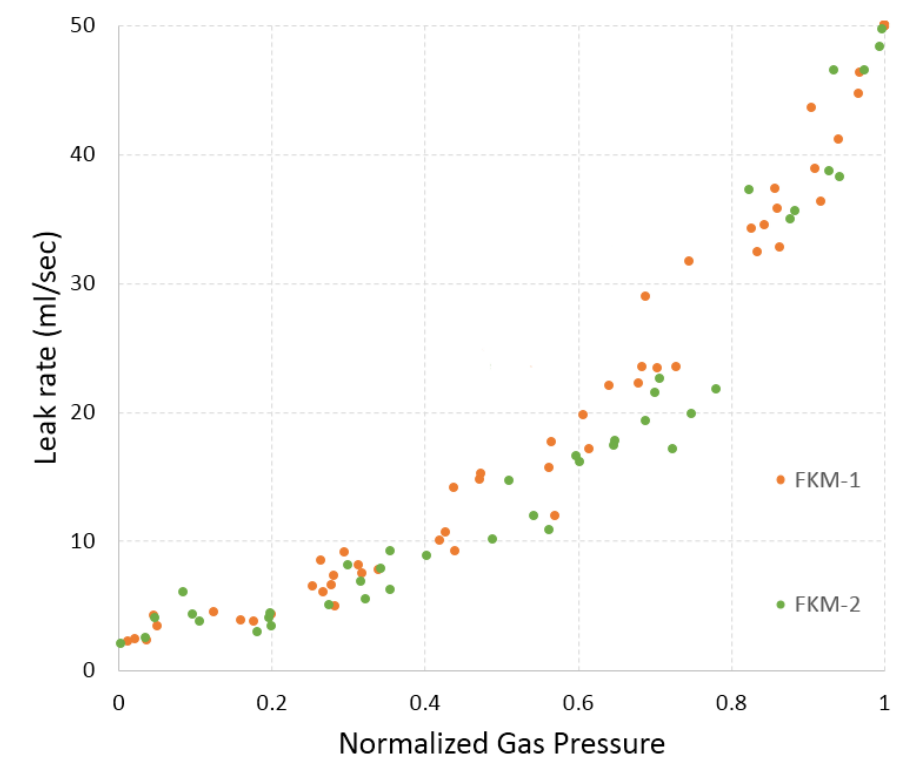

Figure 13 Leak rate v/s normalised gas pressure (see online version for colours)

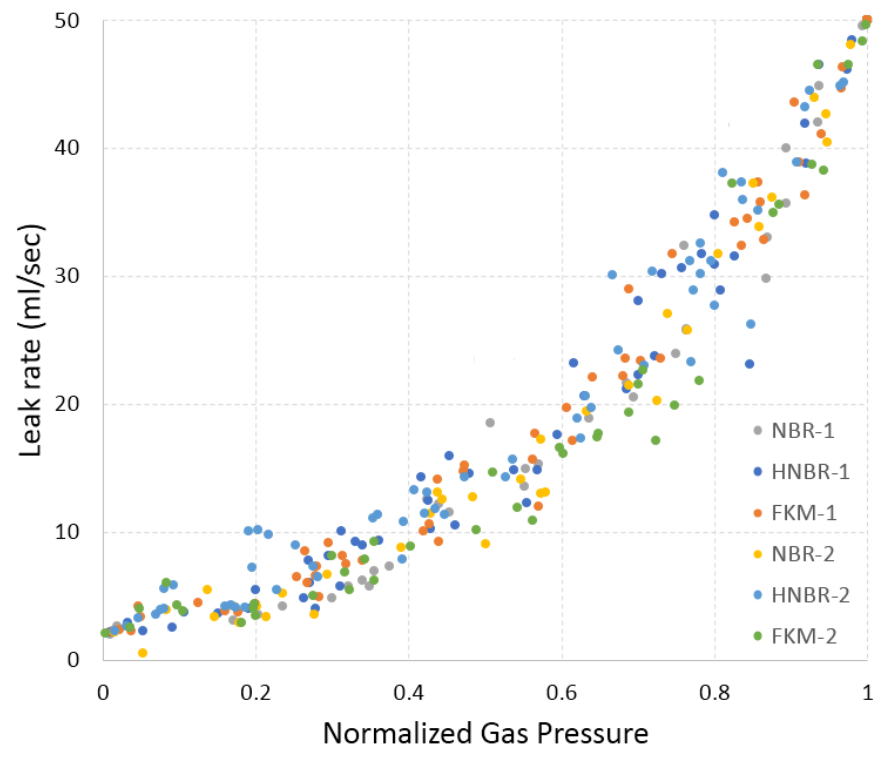




\section{Conclusions}

The present paper discusses the need for experiments that can characterise leaks through elastomer-metal interface. Although, similar experiments were already performed by Liu et al. (2014) and Lorenz and Persson (2009), such experiments lacked in terms of the sealing materials used, the amount of data reported and/or the number of parameters that were varied during the experiments. Experiments thus conducted by varying both sealing load intensity at zero pressure and fluid pressure independently using elastomeric sheets of different materials showed that the leak rate increases with gas pressure and decreases with sealing load intensity at zero pressure. While different materials showed different leak initiation and failure initiation pressures, the material independent behaviour of this leak could be obtained by normalisation of pressure. This shows that leak initiation pressure and the flow characteristics of leak can be captured independently for a given geometry. Similarly, it is also required to identify the geometry independent behaviour of leak to predict failure of seals of different sizes and shapes. This can be accomplished by modelling mechanics of leak using carefully designed hypothesis and more experimentation for verification of the same.

\section{Acknowledgements}

We sincerely thank J.K. Fenner (India) Limited, Madurai for supporting us with necessary elastomeric material during our tests.

\section{References}

Briscoe, B.J., Savvas, T. and Kelly, C.T. (1994) 'Explosive decompression failure' of rubbers: a review of the origins of pneumatic stress induced rupture in elastomers', Rubber Chemistry and Technology, Vol. 67, No. 3, pp.384-416.

Druecke, B., Dussan, V.E.B., Wicks, N. and Hosoi, A.E. (2015) 'Large elastic deformation as a mechanism for soft seal leakage', Journal of Applied Physics, Vol. 117, No. 10, p.104511.

Etsion, I. and Front, I. (1994) 'A model for static sealing performance of end face seals', Tribology Transactions, Vol. 37, No. 1, pp.111-119.

Gillen, K.T., Bernstein, R. and Wilson, M.H. (2005) 'Predicting and confirming the lifetime of orings', Polymer Degradation and Stability, Vol. 87, No. 2, pp.257-270.

Gillen, K.T., Celina, M. and Bernstein, R. (2003) 'Validation of improved methods for predicting long-term elastomeric seal lifetimes from compression stress-relaxation and oxygen consumption techniques', Polymer Degradation and Stability, Vol. 82, No. 1, pp.25-35.

Grelle, T., Wolff, D. and Jaunich, M. (2017) 'Leakage behaviour of elastomer seals under dynamic unloading conditions at low temperatures', Polymer Testing, Vol. 58, pp.219-226.

ISO 23936-2 (2011) Petroleum, Petrochemical and Natural Gas Industries - Non-Metallic Materials in Contact with Media Related to Oil and Gas Production - Part 2: Elastomers.

Ito, M., Noguchi, T., Ueki, H., Takeuchi, K. and Endo, M. (2011) 'Carbon nanotube enables quantum leap in oil recovery', Materials Research Bulletin, Vol. 46, No. 9, pp.1480-1484.

Kirthan, L.J., Hegde, R., Girisha, V.A. and Kumar, R.G. (2016) 'Evaluation of mode 1 stress intensity factor for edge crack using displacement extrapolation method', International Journal of Materials and Structural Integrity, Vol. 10, Nos. 1-3, pp.11-22. 
Liu, J., Li, X., Xu, L. and He, T. (2017) 'Service lifetime estimation of EPDM rubber based on accelerated aging tests', Journal of Materials Engineering and Performance, Vol. 26, No. 4, pp.1735-1740.

Liu, Q., Wang, Z., Lou, Y. and Suo, Z. (2014) 'Elastic leak of a seal', Extreme Mechanics Letters, Vol. 1, pp.54-61.

Lorenz, B. and Persson, B.N.J. (2009) 'Leak rate of seals: comparison of theory with experiment', EPL (Europhysics Letters), Vol. 86, No. 4, p.44006.

Mars, W.V. and Fatemi, A. (2002) 'A literature survey on fatigue analysis approaches for rubber', International Journal of Fatigue, Vol. 24, No. 9, pp.949-961.

Moan, G.D., Coleman, C.E., Price, E.G., Rodgers, D.K. and Sagat, S. (1990) 'Leak-before-break in the pressure tubes of CANDU reactors', International Journal of Pressure Vessels and Piping, Vol. 43, Nos. 1-3, pp.1-21.

Takahashi, Y. (2002) 'Evaluation of leak-before-break assessment methodology for pipes with a circumferential through-wall crack: Part I: stress intensity factor and limit load solutions', International Journal of Pressure Vessels and Piping, Vol. 79, No. 6, pp.385-392.

Weitzel, D.H., Robbins, R.F., Bopp, G.R. and Bjorklund, W.R. (1960) 'Low temperature static seals using elastomers and plastics', Review of Scientific Instruments, Vol. 31, No. 12, pp.1350-1351. 